\title{
A New Reversed Version of a Generalized Sharp Hölder's Inequality and Its Applications
}

\author{
Jingfeng Tian' ${ }^{1}$ and Xi-Mei Hu, ${ }^{1,2}$ \\ ${ }^{1}$ College of Science and Technology, North China Electric Power University, Baoding 071000, China \\ ${ }^{2}$ China Mobile Group Hebei Co., Ltd., Baoding 071000, China
}

Correspondence should be addressed to Xi-Mei Hu; huxm_ncepu@yahoo.cn

Received 10 October 2012; Accepted 10 January 2013

Academic Editor: Pekka Koskela

Copyright (C 2013 J. Tian and X.-M. Hu. This is an open access article distributed under the Creative Commons Attribution License, which permits unrestricted use, distribution, and reproduction in any medium, provided the original work is properly cited.

We present a new reversed version of a generalized sharp Hölder's inequality which is due to Wu and then give a new refinement of Hölder's inequality. Moreover, the obtained result is used to improve the well-known Popoviciu-Vasić inequality. Finally, we establish the time scales version of Beckenbach-type inequality.

\section{Introduction}

The classical Hölder's inequality states that if $a_{k} \geq 0, b_{k} \geq$ $0(k=1,2, \ldots, n), p>1$, and $1 / p+1 / q=1$, then

$$
\sum_{k=1}^{n} a_{k} b_{k} \leq\left(\sum_{k=1}^{n} a_{k}^{p}\right)^{1 / p}\left(\sum_{k=1}^{n} b_{k}^{q}\right)^{1 / q} .
$$

The inequality is reversed for $p<1(p \neq 0)$, (for $p<0$, we assume that $a_{k}, b_{k}>0$ ).

As is well known, Hölder's inequality plays an important role in different branches of modern mathematics such as classical real and complex analysis, probability and statistics, numerical analysis, and qualitative theory of differential equations and their applications. Various refinements, generalizations, and applications of inequality (1) and its series analogues in different areas of mathematics have appeared in the literature. For example, Abramovich et al. [1] presented a new generalization of Hölder's inequality and its reversed version in discrete and integral forms. Ivanković et al. [2] presented the properties of several mappings which have arisen from the Minkowski inequality and then gave some refinements of the Hölder inequality. Liu [3] obtained Hölder's inequality in fuzzy set theory and rough set theory. Nikolova and Varošanec [4] obtained some new refinements of the classical Hölder inequality by using a convex function.
For detailed expositions, the interested reader may consult [1$18]$ and the references therein.

Among various refinements of (1), Hu in [9] established the following interesting sharpness of Hölder's inequality.

Theorem A. Let $p \geq q>0,1 / p+1 / q=1$, let $A_{n}, B_{n} \geq 0$, $\sum_{n} A_{n}^{p}<\infty$, and $\sum_{n} B_{n}^{q}<\infty$, and let $1-e_{n}+e_{m} \geq 0, \sum_{n}\left|e_{n}\right|<$ $\infty$. Then,

$$
\begin{aligned}
\sum_{n} A_{n} B_{n} \leq & \left(\sum_{n} B_{n}^{q}\right)^{1 / q-1 / p} \\
& \times\left\{\left[\left(\sum_{n} B_{n}^{q}\right)\left(\sum_{n} A_{n}^{p}\right)\right]^{2}\right. \\
& -\left[\left(\sum_{n} B_{n}^{q} e_{n}\right)\left(\sum_{n} A_{n}^{p}\right)\right. \\
& \left.\left.-\left(\sum_{n} B_{n}^{q}\right)\left(\sum_{n} A_{n}^{p} e_{n}\right)\right]^{2}\right\}^{1 / 2 p} .
\end{aligned}
$$

In 2007, Wu [18] presented the generalization of Hu's result as follows. 
Theorem B. Let $A_{r} \geq 0, B_{r}>0(r=1,2, \ldots, n)$, let $1-e_{r}+$ $e_{s} \geq 0(r, s=1,2, \ldots, n)$, and let $p \geq q>0, \mu=\min \{1 / p+$ $1 / q, 1\}$. Then,

$$
\begin{aligned}
\sum_{r=1}^{n} A_{r} B_{r} \leq n^{1-\mu}\left(\sum_{r=1}^{n} B_{r}^{q}\right)^{1 / q-1 / p} & \left\{\left[\left(\sum_{r=1}^{n} B_{r}^{q}\right)\left(\sum_{r=1}^{n} A_{r}^{p}\right)\right]^{2}\right. \\
\times & {\left[\left(\sum_{r=1}^{n} B_{r}^{q} e_{r}\right)\left(\sum_{r=1}^{n} A_{r}^{p}\right)\right.} \\
& \left.\left.-\left(\sum_{r=1}^{n} B_{r}^{q}\right)\left(\sum_{r=1}^{n} A_{r}^{p} e_{r}\right)\right]^{2}\right\}^{1 / 2 p} .
\end{aligned}
$$

Theorem C. Let $f(x), g(x)$, and $e(x)$ be integrable functions defined on $[a, b]$ and $f(x) \geq 0, g(x)>0,1-e(x)+e(y) \geq 0$ for all $x, y \in[a, b]$, and let $p \geq q>0,1 / p+1 / q \leq 1$. Then,

$$
\begin{aligned}
& \int_{a}^{b} f(x) g(x) d x \\
& \leq(b-a)^{1-1 / p-1 / q}\left(\int_{a}^{b} g^{q}(x) d x\right)^{1 / q-1 / p} \\
& \times\left[\left(\int_{a}^{b} g^{q}(x) d x \int_{a}^{b} f^{p}(x) d x\right)^{2}\right. \\
& -\left(\int_{a}^{b} g^{q}(x) e(x) d x \int_{a}^{b} f^{p}(x) d x\right. \\
& \left.\left.-\int_{a}^{b} g^{q}(x) d x \int_{a}^{b} f^{p}(x) e(x) d x\right)^{2}\right]^{1 / 2 p} .
\end{aligned}
$$

Recently, Tian in [13] proved the following reversed versions of inequalities (3) and (4).

Theorem D. Let $A_{r}>0, B_{r}>0(r=1,2, \ldots, n)$, let $1-$ $e_{r}+e_{s} \geq 0(r, s=1,2, \ldots, n)$, and let $q<0,1 / p+1 / q \geq 0$, $\mu=\max \{1 / p+1 / q, 1\}, \lambda=\max \{1 / q,-1\}$. Then,

$$
\begin{aligned}
\sum_{r=1}^{n} A_{r} B_{r} & \leq n^{1-\mu}\left(\sum_{r=1}^{n} A_{r}^{p}\right)^{1 / p}\left(\sum_{r=1}^{n} B_{r}^{q}\right)^{1 / q} \\
& \times\left[1-\left(\frac{\sum_{r=1}^{n} A_{r} B_{r} e_{r}}{\sum_{r=1}^{n} A_{r} B_{r}}-\frac{\sum_{r=1}^{n} B_{r}^{q} e_{r}}{\sum_{r=1}^{n} B_{r}^{q}}\right)^{2}\right]^{\lambda / 2} .
\end{aligned}
$$

Theorem E. Let $f(x), g(x)$, and $e(x)$ be integrable functions defined on $[a, b]$ and $f(x)>0, g(x)>0$, and $1-e(x)+e(y) \geq 0$ for all $x, y \in[a, b]$, and let $q<0,1 / p+$ $1 / q \geq 1, \lambda=\max \{-1,1 / q\}$. Then,

$$
\begin{aligned}
\int_{a}^{b} f(x) g(x) d x \geq & (b-a)^{1-1 / p-1 / q} \\
& \times\left(\int_{a}^{b} f^{p}(x) d x\right)^{1 / p}\left(\int_{a}^{b} g^{q}(x) d x\right)^{1 / q} \\
& \times\left[1-\left(\frac{\int_{a}^{b} f(x) g(x) e(x) d x}{\int_{a}^{b} f(x) g(x) d x}\right.\right. \\
& \left.\left.-\frac{\int_{a}^{b} g^{q}(x) e(x) d x}{\int_{a}^{b} g^{q}(x) d x}\right)^{2}\right]^{\lambda / 2}
\end{aligned}
$$

The aim of this paper is to give new reversed versions of (3) and (4). Moreover, two applications of the obtained results are presented. The rest of this paper is organized as follows. In Section 2, we present reversed versions of (3) and (4). Moreover, we give a new refinement of Hölder's inequality. In Section 3, we apply the obtained result to improve the Popoviciu-Vasic inequality. Furthermore, we establish the time scales version of Beckenbach-type inequality.

\section{A New Reversed Version of a Generalized Sharp Hölder's Inequality}

In order to prove the main results, we need the following lemmas.

Lemma 1 (see, e.g., [11, page 12]). Let $A_{k j}>0(j=$ $1,2, \ldots, m, k=1,2, \ldots, n), \sum_{j=1}^{m} 1 / p_{j} \leq 1$. If $p_{1}>0, p_{j}<$ $0(j=2,3, \ldots, m)$, then

$$
\sum_{k=1}^{n} \prod_{j=1}^{m} A_{k j} \geq \prod_{j=1}^{m}\left(\sum_{k=1}^{n} A_{k j}^{p_{j}}\right)^{1 / p_{j}} .
$$

Lemma 2 (see [19, page 12]). If $x>-1, \alpha \geq 1$, or $\alpha<0$, then

$$
(1+x)^{\alpha} \geq 1+\alpha x
$$

The inequality is reversed for $0<\alpha \leq 1$.

Lemma 3 (see [7, page 27]). If $x_{i} \geq 0, \lambda_{i}>0, i=1,2, \ldots, n$, and $0<p \leq 1$, then

$$
\sum_{i=1}^{n} \lambda_{i} x_{i}^{p} \leq\left(\sum_{i=1}^{n} \lambda_{i}\right)^{1-p}\left(\sum_{i=1}^{n} \lambda_{i} x_{i}\right)^{p} .
$$

The inequality is reversed for $p \geq 1$ or $p<0$.

Next, we give a reversed version of inequality (3) as follows. 
Theorem 4. Let $A_{r} \geq 0, B_{r}>0(r=1,2, \ldots, n)$, let $1-e_{r}+$ $e_{s} \geq 0(r, s=1,2, \ldots, n)$, and let $q<0, p>0, \rho=\max \{1 / p+$ $1 / q, 1\}$. Then,

$$
\begin{aligned}
\sum_{r=1}^{n} A_{r} B_{r} \geq n^{1-\rho}\left(\sum_{r=1}^{n} A_{r}^{p}\right)^{1 / p-1 / q} \\
\times\left\{\left[\left(\sum_{r=1}^{n} A_{r}^{p}\right)\left(\sum_{r=1}^{n} B_{r}^{q}\right)\right]^{2}\right. \\
-\left[\left(\sum_{r=1}^{n} A_{r}^{p} e_{r}\right)\left(\sum_{r=1}^{n} B_{r}^{q}\right)\right. \\
\left.\left.-\left(\sum_{r=1}^{n} A_{r}^{p}\right)\left(\sum_{r=1}^{n} B_{r}^{q} e_{r}\right)\right]^{2}\right\}^{1 / 2 q} .
\end{aligned}
$$

Proof. We first consider the case (I) $1 / p+1 / q \leq 1$. On one hand, performing some simple computations, we have

$$
\begin{aligned}
\sum_{r=1}^{n} A_{r} & B_{r} \sum_{s=1}^{n} A_{s} B_{s}\left(1-e_{r}+e_{s}\right) \\
= & \sum_{s=1}^{n} \sum_{r=1}^{n} A_{r} B_{r} A_{s} B_{s}-\sum_{s=1}^{n} \sum_{r=1}^{n} A_{r} B_{r} A_{s} B_{s} e_{r} \\
& +\sum_{s=1}^{n} \sum_{r=1}^{n} A_{r} B_{r} A_{s} B_{s} e_{s} \\
= & \left(\sum_{k=1}^{n} A_{k} B_{k}\right)^{2} .
\end{aligned}
$$

On the other hand, by using inequality (9), we have

$$
\begin{aligned}
\sum_{r=1}^{n} A_{r} & B_{r} \sum_{s=1}^{n} A_{s} B_{s}\left(1-e_{r}+e_{s}\right)^{1 / p+1 / q} \\
= & \sum_{r=1}^{n} \sum_{s=1}^{n} A_{r} B_{r} A_{s} B_{s}\left(1-e_{r}+e_{s}\right)^{1 / p+1 / q} \\
\leq & \left(\sum_{r=1}^{n} \sum_{s=1}^{n} A_{r} B_{r} A_{s} B_{s}\right)^{1-1 / p-1 / q} \\
& \times\left(\sum_{r=1}^{n} \sum_{s=1}^{n} A_{r} B_{r} A_{s} B_{s}\left(1-e_{r}+e_{s}\right)\right)^{1 / p+1 / q} \\
= & \left(\sum_{r=1}^{n} \sum_{s=1}^{n} A_{r} B_{r} A_{s} B_{s}\right)^{1-1 / p-1 / q} \\
& \times\left(\sum_{r=1}^{n} \sum_{s=1}^{n} A_{r} B_{r} A_{s} B_{s}-\sum_{r=1}^{n} \sum_{s=1}^{n} A_{r} B_{r} A_{s} B_{s} e_{r}\right. \\
& \left.+\sum_{r=1}^{n} \sum_{s=1}^{n} A_{r} B_{r} A_{s} B_{s} e_{s}\right)^{1 / p+1 / q}
\end{aligned}
$$

$$
\begin{aligned}
= & \left(\sum_{r=1}^{n} \sum_{s=1}^{n} A_{r} B_{r} A_{s} B_{s}\right)^{1-1 / p-1 / q} \\
& \times\left(\sum_{r=1}^{n} \sum_{s=1}^{n} A_{r} B_{r} A_{s} B_{s}\right)^{1 / p+1 / q} \\
= & \sum_{r=1}^{n} \sum_{s=1}^{n} A_{r} B_{r} A_{s} B_{s}=\left(\sum_{r=1}^{n} A_{r} B_{r}\right)^{2} .
\end{aligned}
$$

By using inequality (7), we have

$$
\sum_{r=1}^{n} A_{r} B_{r} \sum_{s=1}^{n} A_{s} B_{s}\left(1-e_{r}+e_{s}\right)^{1 / p+1 / q}
$$

$$
\geq \sum_{r=1}^{n} A_{r} B_{r}\left(\sum_{s=1}^{n} A_{s}^{p}\left(1-e_{r}+e_{s}\right)\right)^{1 / p}
$$$$
\times\left(\sum_{s=1}^{n} B_{s}^{q}\left(1-e_{r}+e_{s}\right)\right)^{1 / q}
$$

$$
=\sum_{r=1}^{n}\left[\left(\sum_{s=1}^{n} A_{r}^{p} A_{s}^{p}\left(1-e_{r}+e_{s}\right)\right)^{1 / p-1 / q}\right.
$$

$$
\begin{aligned}
& \times\left(\sum_{s=1}^{n} A_{r}^{p} B_{s}^{q}\left(1-e_{r}+e_{s}\right)\right)^{1 / q} \\
& \left.\times\left(\sum_{s=1}^{n} B_{r}^{q} A_{s}^{p}\left(1-e_{r}+e_{s}\right)\right)^{1 / q}\right] .
\end{aligned}
$$

Consequently, according to $(1 / p-1 / q)+1 / q+1 / q \leq 1$, by using inequality (7) on the right side of (13), we observe that

$$
\begin{aligned}
\sum_{r=1}^{n} A_{r} B_{r} \sum_{s=1}^{n} A_{s} B_{s}\left(1-e_{r}+e_{s}\right)^{1 / p+1 / q} \\
\geq\left(\sum_{r=1}^{n} \sum_{s=1}^{n} A_{r}^{p} A_{s}^{p}\left(1-e_{r}+e_{s}\right)\right)^{1 / p-1 / q} \\
\quad \times\left(\sum_{r=1}^{n} \sum_{s=1}^{n} A_{r}^{p} B_{s}^{q}\left(1-e_{r}+e_{s}\right)\right)^{1 / q} \\
\quad \times\left(\sum_{r=1}^{n} \sum_{s=1}^{n} B_{r}^{q} A_{s}^{p}\left(1-e_{r}+e_{s}\right)\right)^{1 / q} \\
=\left(\sum_{r=1}^{n} A_{r}^{p}\right)^{2 / p-2 / q}
\end{aligned}
$$




$$
\begin{gathered}
\times\left[\left(\sum_{r=1}^{n} A_{r}^{p} \sum_{s=1}^{n} B_{s}^{q}-\sum_{r=1}^{n} A_{r}^{p} e_{r} \sum_{s=1}^{n} B_{s}^{q}+\sum_{r=1}^{n} A_{r}^{p} \sum_{s=1}^{n} B_{s}^{q} e_{s}\right)\right. \\
\left.\times\left(\sum_{r=1}^{n} B_{r}^{q} \sum_{s=1}^{n} A_{s}^{p}-\sum_{r=1}^{n} B_{r}^{q} e_{r} \sum_{s=1}^{n} A_{s}^{p}+\sum_{r=1}^{n} B_{r}^{q} \sum_{s=1}^{n} A_{s}^{p} e_{s}\right)\right]^{1 / q} \\
=\left(\sum_{r=1}^{n} A_{r}^{p}\right)^{2 / p-2 / q} \\
\times\left\{\left[\left(\sum_{r=1}^{n} A_{r}^{p}\right)\left(\sum_{r=1}^{n} B_{r}^{q}\right)\right]^{2}\right. \\
\left.-\left[\left(\sum_{r=1}^{n} A_{r}^{p} e_{r}\right)\left(\sum_{r=1}^{n} B_{r}^{q}\right)-\left(\sum_{r=1}^{n} A_{r}^{p}\right)\left(\sum_{r=1}^{n} B_{r}^{q} e_{r}\right)\right]^{2}\right\}^{1 / q} .
\end{gathered}
$$

Combining inequalities (12) and (14) leads to inequality (10) immediately.

Secondly, we consider the case (II) $1 / p+1 / q \geq 1$. Let $1 / p+1 / q=t(t \geq 1)$, which implies that $1 / p t+1 / q t=1$. From Hölder's inequality and (7), we have

$$
\begin{aligned}
\sum_{r=1}^{n} A_{r} B_{r} \sum_{s=1}^{n} A_{s} B_{s}\left(1-e_{r}+e_{s}\right) & \\
= & \sum_{r=1}^{n} A_{r} B_{r} \sum_{s=1}^{n} A_{s} B_{s}\left(1-e_{r}+e_{s}\right)^{1 / p t+1 / q t} \\
\geq & \sum_{r=1}^{n} A_{r} B_{r}\left[\left(\sum_{s=1}^{n} A_{s}^{p t}\left(1-e_{r}+e_{s}\right)\right)^{1 / p t}\right. \\
= & \left.\sum_{r=1}^{n}\left[\left(\sum_{s=1}^{n} A_{r}^{p t} A_{s}^{p t}\left(1-e_{r}+e_{s}\right)\right)^{q t}\left(1-e_{r}+e_{s}\right)\right)^{1 / q t}\right] \\
& \times\left(\sum_{s=1}^{n} B_{r}^{q t} A_{s}^{p t}\left(1-e_{r}+e_{s}\right)\right)^{1 / q t} \\
\geq & \times\left(\sum_{r=1}^{n} \sum_{s=1}^{n} \sum_{s=1}^{n} A_{r}^{p t} B_{r}^{q t} A_{s}^{p t} B_{s}^{q t}\left(1-e_{r}^{p t}\left(1-e_{r}+e_{s}\right)\right)^{1 / p t-1 / q t}\right. \\
& \left.\times\left(\sum_{s=1}^{n} A_{r}^{p t} B_{s}^{q t}\left(1-e_{r}+e_{s}\right)\right)^{1 / q t}\right] \\
& \times
\end{aligned}
$$

Additionally, using Lemma 3 together with $t \geq 1$, we find $\left(\sum_{r=1}^{n} \sum_{s=1}^{n} A_{r}^{p t} A_{s}^{p t}\left(1-e_{r}+e_{s}\right)\right)^{1 / p t-1 / q t}$

$$
\begin{aligned}
& \times\left(\sum_{r=1}^{n} \sum_{s=1}^{n} B_{r}^{q t} A_{s}^{p t}\left(1-e_{r}+e_{s}\right)\right)^{1 / q t} \\
& \times\left(\sum_{r=1}^{n} \sum_{s=1}^{n} A_{r}^{p t} B_{s}^{q t}\left(1-e_{r}+e_{s}\right)\right)^{1 / q t} \\
& \geq\left(\sum_{r=1}^{n} \sum_{s=1}^{n}\left(1-e_{r}+e_{s}\right)\right)^{(1-t)(1 / p t-1 / q t)} \\
& \times\left(\sum_{r=1}^{n} \sum_{s=1}^{n} A_{r}^{p} A_{s}^{p}\left(1-e_{r}+e_{s}\right)\right)^{1 / p-1 / q} \\
& \times\left(\sum_{r=1}^{n} \sum_{s=1}^{n}\left(1-e_{r}+e_{s}\right)\right)^{(1-t) / q t}
\end{aligned}
$$$$
\times\left(\sum_{r=1}^{n} \sum_{s=1}^{n} B_{r}^{q} A_{s}^{p}\left(1-e_{r}+e_{s}\right)\right)^{1 / q}
$$$$
\times\left(\sum_{r=1}^{n} \sum_{s=1}^{n}\left(1-e_{r}+e_{s}\right)\right)^{(1-t) / q t}
$$$$
\times\left(\sum_{r=1}^{n} \sum_{s=1}^{n} A_{r}^{p} B_{s}^{q}\left(1-e_{r}+e_{s}\right)\right)^{1 / q}
$$$$
=\left(\sum_{r=1}^{n} \sum_{s=1}^{n}\left(1-e_{r}+e_{s}\right)\right)^{1-t}
$$$$
\times\left(\sum_{r=1}^{n} \sum_{s=1}^{n} A_{r}^{p} A_{s}^{p}\left(1-e_{r}+e_{s}\right)\right)^{1 / p-1 / q}
$$$$
\times\left(\sum_{r=1}^{n} \sum_{s=1}^{n} B_{r}^{q} A_{s}^{p}\left(1-e_{r}+e_{s}\right)\right)^{1 / q}
$$$$
\times\left(\sum_{r=1}^{n} \sum_{s=1}^{n} A_{r}^{p} B_{s}^{q}\left(1-e_{r}+e_{s}\right)\right)^{1 / q}
$$$$
=n^{2-2 t}\left(\sum_{r=1}^{n} A_{r}^{p}\right)^{2 / p-2 / q}
$$$$
\times\left(\sum_{r=1}^{n} B_{r}^{q} \sum_{s=1}^{n} A_{s}^{p}-\sum_{r=1}^{n} B_{r}^{q} e_{r} \sum_{s=1}^{n} A_{s}^{p}+\sum_{r=1}^{n} B_{r}^{q} \sum_{s=1}^{n} A_{s}^{p} e_{s}\right)^{1 / q}
$$$$
\times\left(\sum_{r=1}^{n} A_{r}^{p} \sum_{s=1}^{n} B_{s}^{q}-\sum_{r=1}^{n} A_{r}^{p} e_{r} \sum_{s=1}^{n} B_{s}^{q}+\sum_{r=1}^{n} A_{r}^{p} \sum_{s=1}^{n} B_{s}^{q} e_{s}\right)^{1 / q}
$$$$
=n^{2(1-1 / p-1 / q)}\left(\sum_{r=1}^{n} A_{r}^{p}\right)^{2 / p-2 / q}
$$$$
\times\left\{\left[\left(\sum_{r=1}^{n} A_{r}^{p}\right)\left(\sum_{r=1}^{n} B_{r}^{q}\right)\right]^{2}\right.
$$

$$
\left.-\left[\left(\sum_{r=1}^{n} A_{r}^{p} e_{r}\right)\left(\sum_{r=1}^{n} B_{r}^{q}\right)-\left(\sum_{r=1}^{n} A_{r}^{p}\right)\left(\sum_{r=1}^{n} B_{r}^{q} e_{r}\right)\right]^{2}\right\}^{1 / q} .
$$


Combining inequalities (11), (15), and (16) leads to inequality (10) immediately.

The proof of Theorem 4 is completed.

From Theorem 4 and Lemma 2, we obtain the refinement of Hölder's inequality (1) as follows.

Corollary 5. Let $A_{r}>0, B_{r}>0(r=1,2, \ldots, n)$, let $1-$ $e_{r}+e_{s} \geq 0(r, s=1,2, \ldots, n)$, and let $q<0, p>0$, and $\rho=\max \{1 / p+1 / q, 1\}$. Then,

$$
\begin{aligned}
\sum_{r=1}^{n} A_{r} B_{r} \geq & n^{1-\rho}\left(\sum_{r=1}^{n} A_{r}^{p}\right)^{1 / p}\left(\sum_{r=1}^{n} B_{r}^{q}\right)^{1 / q} \\
& \times\left[1-\frac{1}{2 q}\left(\frac{\sum_{r=1}^{n} B_{r}^{q} e_{r}}{\sum_{k=1}^{n} B_{r}^{q}}-\frac{\sum_{r=1}^{n} A_{r}^{p} e_{r}}{\sum_{r=1}^{n} A_{r}^{p}}\right)^{2}\right] .
\end{aligned}
$$

Proof. Since

$$
\left|\frac{\sum_{r=1}^{n} B_{r}^{q} e_{r}}{\sum_{k=1}^{n} B_{r}^{q}}-\frac{\sum_{r=1}^{n} A_{r}^{p} e_{r}}{\sum_{r=1}^{n} A_{r}^{p}}\right|<1,
$$

by using Lemma 2 and Theorem 4, we obtain the assertion of the corollary. The proof of Corollary 5 is completed.

Now, we give a reversed version of inequality (4) as follows.

Theorem 6. Let $f(x), g(x)$, and $e(x)$ be integrable functions defined on $[a, b]$ and $f(x), g(x)>0,1-e(x)+e(y) \geq 0$ for all $x, y \in[a, b]$, and let $q<0,1 / p+1 / q \geq 1$. Then,

$$
\begin{gathered}
\int_{a}^{b} f(x) g(x) d x \\
\geq(b-a)^{1-1 / p-1 / q}\left(\int_{a}^{b} f^{p}(x) d x\right)^{1 / p-1 / q} \\
\times\left[\left(\int_{a}^{b} f^{p}(x) d x \int_{a}^{b} g^{q}(x) d x\right)^{2}\right. \\
-\left(\int_{a}^{b} f^{p}(x) e(x) d x \int_{a}^{b} g^{q}(x) d x\right. \\
\left.\left.-\int_{a}^{b} f^{p}(x) d x \int_{a}^{b} g^{q}(x) e(x) d x\right)^{2}\right]^{1 / 2 q} .
\end{gathered}
$$

Proof. For any positive integer $n$, we choose an equidistant partition of $[a, b]$ as follows:

$$
\begin{aligned}
& a<a+\frac{b-a}{n}<\cdots<a+\frac{b-a}{n} k<\cdots<a+\frac{b-a}{n}(n-1)<b, \\
& x_{k}=a+\frac{b-a}{n} k, \quad \Delta x_{k}=\frac{b-a}{n}, \quad k=0,1,2, \ldots, n .
\end{aligned}
$$

Applying Theorem 4, we obtain the following inequality:

$$
\begin{gathered}
\sum_{k=1}^{n} f\left(x_{k}\right) g\left(x_{k}\right) \\
\geq n^{1-1 / p-1 / q}\left(\sum_{k=1}^{n} f^{p}\left(x_{k}\right)\right)^{1 / p-1 / q} \\
\times\left\{\left[\left(\sum_{k=1}^{n} f^{p}\left(x_{k}\right)\right)\left(\sum_{k=1}^{n} g^{q}\left(x_{k}\right)\right)\right]^{2}\right. \\
-\left[\left(\sum_{k=1}^{n} f^{p}\left(x_{k}\right) e\left(x_{k}\right)\right)\left(\sum_{k=1}^{n} g^{q}\left(x_{k}\right)\right)\right. \\
-\left(\sum_{k=1}^{n} f^{p}\left(x_{k}\right)\right) \\
\left.\times\left(\sum_{k=1}^{n} g^{q}\left(x_{k}\right) e\left(x_{k}\right)\right)\right]^{2},
\end{gathered}
$$

equivalently

$$
\begin{gathered}
\sum_{k=1}^{n} f\left(x_{k}\right) g\left(x_{k}\right) \frac{b-a}{n} \\
\geq(b-a)^{1-1 / p-1 / q}\left(\sum_{k=1}^{n} f^{p}\left(x_{k}\right) \frac{b-a}{n}\right)^{1 / p-1 / q} \\
\times\left\{\left[\left(\sum_{k=1}^{n} f^{p}\left(x_{k}\right) \frac{b-a}{n}\right)\left(\sum_{k=1}^{n} g^{q}\left(x_{k}\right) \frac{b-a}{n}\right)\right]^{2}\right. \\
-\left[\left(\sum_{k=1}^{n} f^{p}\left(x_{k}\right) e\left(x_{k}\right) \frac{b-a}{n}\right)\left(\sum_{k=1}^{n} g^{q}\left(x_{k}\right) \frac{b-a}{n}\right)\right. \\
-\left(\sum_{k=1}^{n} f^{p}\left(x_{k}\right) \frac{b-a}{n}\right) \\
\left.\left.\times\left(\sum_{k=1}^{n} g^{q}\left(x_{k}\right) e\left(x_{k}\right) \frac{b-a}{n}\right)\right]^{2}\right\}^{1 / 2 q} \cdot
\end{gathered}
$$

In view of the hypotheses that $f(x), g(x)$, and $e(x)$ are positive Riemann integrable functions on $[a, b]$, we conclude that $f^{p}(x), g^{q}(x)$, and $g^{q}(x) e(x)$ are also integrable on $[a, b]$. Passing the limit as $n \rightarrow \infty$ in both sides of inequality (22), we obtain inequality (19). The proof of Theorem 6 is completed. 
Remark 7. Making similar technique as in the proof of Corollary 5, from Theorem 6 we obtain

$$
\begin{aligned}
\int_{a}^{b} f(x) g(x) d x \\
\geq(b-a)^{1-1 / p-1 / q}\left(\int_{a}^{b} f^{p}(x) d x\right)^{1 / p}\left(\int_{a}^{b} g^{q}(x) d x\right)^{1 / q} \\
\quad \times\left[1-\frac{1}{2 q}\left(\frac{\int_{a}^{b} f^{p}(x) e(x) d x}{\int_{a}^{b} f^{p}(x) d x}-\frac{\int_{a}^{b} g^{q}(x) e(x) d x}{\int_{a}^{b} g^{q}(x) d x}\right)^{2}\right] .
\end{aligned}
$$

\section{Applications}

In this section, we show two applications of the inequalities newly obtained in Section 2.

Firstly, we provide an application of the obtained result to improve the Popoviciu-Vasić inequality. In 1956, Aczél [20] established the following inequality.

Theorem F. If $a_{i}, b_{i}(i=1,2, \ldots, n)$ are positive numbers such that $a_{1}^{2}-\sum_{i=2}^{n} a_{i}^{2}>0$ or $b_{1}^{2}-\sum_{i=2}^{n} b_{i}^{2}>0$, then

$$
\left(a_{1}^{2}-\sum_{i=2}^{n} a_{i}^{2}\right)\left(b_{1}^{2}-\sum_{i=2}^{n} b_{i}^{2}\right) \leq\left(a_{1} b_{1}-\sum_{i=2}^{n} a_{i} b_{i}\right)^{2} .
$$

Inequality (24) is the well-known Aczél's inequality, which has many applications in the theory of functional equations in non-Euclidean geometry. Due to the importance of Aczél's inequality, this inequality has been given considerable attention by mathematicians and has motivated a large number of research papers involving different proofs, various generalizations, improvements and applications (see, e.g., [21-24] and the references therein).

One of the most important results in the references mentioned above is the exponential generalization of (24) asserted by Theorem G.

Theorem G. Let $p$ and $q$ be real numbers such that $p, q \neq 0$ and $1 / p+1 / q=1$, and let $a_{i}, b_{i}(i=1,2, \ldots, n)$ be positive numbers such that $a_{1}^{p}-\sum_{i=2}^{n} a_{i}^{p}>0$ and $b_{1}^{q}-\sum_{i=2}^{n} b_{i}^{q}>0$. Then, for $p>1$, one has

$$
\left(a_{1}^{p}-\sum_{i=2}^{n} a_{i}^{p}\right)^{1 / p}\left(b_{1}^{q}-\sum_{i=2}^{n} b_{i}^{q}\right)^{1 / q} \leq a_{1} b_{1}-\sum_{i=2}^{n} a_{i} b_{i} .
$$

If $p<1(p \neq 0)$, one has the reverse inequality.

Remark 8 . The case $p>1$ of Theorem $\mathrm{G}$ was proved by Popoviciu [21]. The case $p<1$ was given in [24] by Vasić and Pečarić.

Now, we give a refinement of inequality (25) by Theorem 4 and Theorem B.
Theorem 9. Let $a_{i}, b_{i} \geq 0, a_{1}^{p}-\sum_{i=2}^{n} a_{i}^{p}>0$, and $b_{1}^{q}-\sum_{i=2}^{n} b_{i}^{q}>$ 0 , let $1-e_{i}+e_{j} \geq 0(i, j=1,2, \ldots, n)$, and let $\mu=\min \{1 / p+$ $1 / q, 1\}, \rho=\max \{1 / p+1 / q, 1\}$. Then, for $p \geq q>0$, one has

$$
\begin{aligned}
& \left(a_{1}^{p}-\sum_{i=2}^{n} a_{i}^{p}\right)^{1 / p}\left(b_{1}^{q}-\sum_{i=2}^{n} b_{i}^{q}\right)^{1 / q} \\
& \leq n^{1-\mu} \times b_{1}^{1-q / p} \\
& \quad \times\left\{b_{1}^{2 q} a_{1}^{2 p}\right. \\
& -\left[a_{1}^{p}\left(b_{1}^{q} e_{1}+\sum_{i=2}^{n} b_{i}^{q}\left(e_{i}-e_{1}\right)\right)\right. \\
& -\sum_{i=2}^{n} a_{i} b_{i} .
\end{aligned}
$$

If $q<0, p>0$, one has

$$
\begin{gathered}
\left(a_{1}^{p}-\sum_{i=2}^{n} a_{i}^{p}\right)^{1 / p}\left(b_{1}^{q}-\sum_{i=2}^{n} b_{i}^{q}\right)^{1 / q} \\
\geq n^{1-\rho} \times a_{1}^{1-p / q} \\
\quad \times\left\{a_{1}^{2 p} b_{1}^{2 q}\right. \\
-\left[b_{1}^{q}\left(a_{1}^{p} e_{1}+\sum_{i=2}^{n} a_{i}^{p}\left(e_{i}-e_{1}\right)\right)\right. \\
-\sum_{i=2}^{n} a_{i} b_{i} .
\end{gathered}
$$

Proof. By substituting

$$
\begin{aligned}
& A_{1}^{p} \longrightarrow a_{1}^{p}-\sum_{i=2}^{n} a_{i}^{p}, \quad B_{1}^{q} \longrightarrow b_{1}^{q}-\sum_{i=2}^{n} b_{i}^{q}, \\
& A_{i} \longrightarrow a_{i}, \quad B_{i} \longrightarrow b_{i} \quad(i=2,3, \ldots, n),
\end{aligned}
$$

in (3) and (10), respectively, we get Theorem 9. 
Remark 10. Let $a_{1} \neq 0, b_{1} \neq 0$, and let $1 / p+1 / q=1$. If $p \geq q>$ 0 , then we conclude from Theorem 9:

$$
\begin{gathered}
\left(a_{1}^{p}-\sum_{i=2}^{n} a_{i}^{p}\right)^{1 / p}\left(b_{1}^{q}-\sum_{i=2}^{n} b_{i}^{q}\right)^{1 / q} \\
\leq a_{1} b_{1}\left\{1-\left(\frac{b_{1}^{q} e_{1}+\sum_{i=2}^{n} b_{i}^{q}\left(e_{i}-e_{1}\right)}{b_{1}^{q}}\right.\right. \\
\left.\left.-\frac{a_{1}^{p} e_{1}+\sum_{i=2}^{n} a_{i}^{p}\left(e_{i}-e_{1}\right)}{a_{1}^{p}}\right)^{2}\right\}^{1 / 2 q} \\
-\sum_{i=2}^{n} a_{i} b_{i} .
\end{gathered}
$$

Inequality (29) is reversed for $q<0$.

Next, we are to establish the time scales version of Beckenbach-type inequality which is due to Wang [25]. In 1983, Wang [25] established the following Beckenbach-type inequality.

Theorem H. Let $f(x)$, and $g(x)$ be positive integrable functions defined on $[s, t]$, and let $1 / p+1 / q=1$. If $0<p<1$, then, for any of the positive numbers: $a, b$, or $c$, the inequality

$$
\frac{\left(a+c \int_{s}^{t} k^{p}(x) d x\right)^{1 / p}}{b+c \int_{s}^{t} k(x) g(x) d x} \geq \frac{\left(a+c \int_{s}^{t} f^{p}(x) d x\right)^{1 / p}}{b+c \int_{s}^{t} f(x) g(x) d x}
$$

holds, where $k(x)=(a g(x) / b)^{q / p}$. The sign of inequality in (30) is reversed if $p>1$.

In order to present the time scales version of (30), we recall the following concepts related to the notion of time scales. A time scale $\mathbb{T}$ is an arbitrary nonempty closed subset of the real numbers $\mathbb{R}$. The forward jump operator and the backward jump operator are defined by

$$
\sigma(t):=\inf \{s \in \mathbb{T}: s>t\}, \quad \rho(t):=\sup \{s \in \mathbb{T}: s<t\},
$$

(supplemented by inf $\emptyset=\sup \mathbb{T}$ and $\sup \emptyset=\inf \mathbb{T}$ ). A point $t \in \mathbb{T}$ is called right scattered, right dense, left scattered, and left dense if $\sigma(t)>t, \sigma(t)=t, \rho(t)<t$, and $\rho(t)=t$ hold, respectively.

A function $f: \mathbb{T} \rightarrow \mathbb{R}$ is said to be rd-continuous if it is continuous at each right dense point and if the left-sided limit exists at every left dense point. The set of all rd-continuous functions is denoted by $C_{\mathrm{rd}}[\mathbb{T}, \mathbb{R}]$.

$$
\begin{aligned}
& \text { Let } \\
& \mathbb{T}^{k}:= \begin{cases}\mathbb{T}-m, & \text { if } \mathbb{T} \text { has left scattered point in } M, \\
\mathbb{T}, & \text { otherwise. }\end{cases}
\end{aligned}
$$

Let $f$ be a function defined on $\mathbb{R}$. Then $f$ is called differentiable at $t \in \mathbb{T}^{k}$, with (delta) derivative $f^{\Delta}(t)$ if given $\epsilon>0$; there exists a neighbourhood $\mathbb{N}$ of $t$ such that

$$
\left|f(\sigma(t))-f(s)-f^{\Delta}(t)(\sigma(t)-s)\right| \leq \epsilon|\sigma(t)-s|
$$

for all $s \in \mathbb{N}$.
Remark 11. If $\mathbb{T}=\mathbb{R}$, then $f^{\Delta}(t)$ becomes the usual derivative; that is, $f^{\Delta}(t)=f^{\prime}(t)$. If $\mathbb{T}=\mathbb{Z}$, then $f^{\Delta}(t)$ reduces to the usual forward difference; that is, $f^{\Delta}(t)=\Delta f(t)$.

A function $F: \mathbb{T} \rightarrow \mathbb{R}$ is called an antiderivative of $f:$ $\mathbb{T} \rightarrow \mathbb{R}$ provided that $F^{\Delta}=f(t)$ holds for all $t \in \mathbb{T}$. In this case, we define the integral of $f$ by

$$
\int_{s}^{t} f(\tau) \Delta \tau=F(t)-F(s),
$$

where $s, t \in \mathbb{T}$.

Remark 12. If $\mathbb{T}=\mathbb{R}$, then the time scale integral is an ordinary integral. If $\mathbb{T}=\mathbb{Z}$, then the time-scale integral is a sum.

For more details on time scales theory, the readers may consult [26-29] and the references therein. Now, we present the time scales version of (30) by using Corollary 5 .

Theorem 13. Let $f(x), g(x)$, and $h(x) \in C_{r d}([s, t],[0,+\infty))$, where $C_{r d}([s, t],[0,+\infty))$ denotes the set of $r d$-continuous functions defined by $C_{r d}([s, t],[0,+\infty))=\{\alpha \mid \alpha:[s, t] \rightarrow$ $[0,+\infty)$ and $\alpha(t)$ is an $r d$-continuous function $\}$ and let $1 / p+$ $1 / q=1$. If $p>1$, then, for any of the positive numbers $a, b$, or $c$, the inequality

$$
\begin{aligned}
& \frac{\left(a+c \int_{s}^{t} h(x) k^{p}(x) \Delta x\right)^{1 / p}}{b+c \int_{s}^{t} h(x) k(x) g(x) \Delta x} \\
& \leq \frac{\left(a+c \int_{s}^{t} h(x) f^{p}(x) \Delta x\right)^{1 / p}}{b+c \int_{s}^{t} h(x) f(x) g(x) \Delta x} \\
& \times\left[1-\frac{1}{2 q}\left(\frac{c \int_{s}^{t} h(x) f^{p}(x) \Delta x}{a+c \int_{s}^{t} h(x) f^{p}(x) \Delta x}\right.\right. \\
& \left.\left.-\frac{c \int_{s}^{t} h(x) g^{q}(x) \Delta x}{a^{-q / p} b^{q}+c \int_{s}^{t} h(x) g^{q}(x) \Delta x}\right)^{2}\right]
\end{aligned}
$$

holds, where $k(x)=(a g(x) / b)^{q / p}$. The sign of inequality in (35) is reversed if $0<p<1$.

Proof. We only consider the case $0<p<1$. Noting that $1+$ $q / p=q$, the left-hand side of (35) becomes

$$
\begin{gathered}
\frac{\left[a+c \int_{s}^{t} h(x)(a g(x) / b)^{q} \Delta x\right]^{1 / p}}{b+c \int_{s}^{t} h(x)(a g(x) / b)^{q / p} g(x) \Delta x} \\
=\frac{(a / b)^{q / p}\left[a(b / a)^{q}+c \int_{s}^{t} h(x) g^{q}(x) \Delta x\right]^{1 / p}}{(a / b)^{q / p}\left[b(b / a)^{q / p}+c \int_{s}^{t} h(x) g^{q}(x) \Delta x\right]} \\
=\left(a^{-q / p} b^{q}+c \int_{s}^{t} h(x) g^{q}(x) \Delta x\right)^{-1 / q} .
\end{gathered}
$$


On the other hand, by using Hölder's inequality and inequality (17) for $e_{1}=0, e_{2}=1$, we obtain

$$
\begin{aligned}
& b+c \int_{s}^{t} h(x) f(x) g(x) \Delta x \\
& \geq b+c\left(\int_{s}^{t} h(x) f^{p}(x) \Delta x\right)^{1 / p} \\
& \times\left(\int_{s}^{t} h(x) g^{q}(x) \Delta x\right)^{1 / q} \\
& =a^{1 / p}\left(b a^{-1 / p}\right)+\left(c \int_{s}^{t} h(x) f^{p}(x) \Delta x\right)^{1 / p} \\
& \times\left(c \int_{s}^{t} h(x) g^{q}(x) \Delta x\right)^{1 / q} \\
& \geq\left(a+c \int_{s}^{t} h(x) f^{p}(x) \Delta x\right)^{1 / p} \\
& \times\left(a^{-q / p} b^{q}+c \int_{s}^{t} h(x) g^{q}(x) \Delta x\right)^{1 / q} \\
& \times\left[1-\frac{1}{2 q}\left(\frac{c \int_{s}^{t} h(x) f^{p}(x) \Delta x}{a+c \int_{s}^{t} h(x) f^{p}(x) \Delta x}\right.\right. \\
& \left.\left.-\frac{c \int_{s}^{t} h(x) g^{q}(x) \Delta x}{a^{-q / p} b^{q}+c \int_{s}^{t} h(x) g^{q}(x) \Delta x}\right)^{2}\right] .
\end{aligned}
$$

Combining inequalities (36) and (38) yields inequality (35). The proof of Theorem 13 is completed.

In (35), taking $c \int_{s}^{t} h(x) f^{p}(x) \Delta x /\left(a+c \int_{s}^{t} h(x) f^{p}(x) \Delta x\right)$ $=c \int_{s}^{t} h(x) g^{q}(x) \Delta x /\left(a^{-q / p} b^{q}+c \int_{s}^{t} h(x) g^{q}(x) \Delta x\right)$, from Theorem 13 we obtain the time scales version of Beckenbachtype inequality as follows.

Corollary 14. Let $f(x), g(x)$, and $h(x) \in C_{r d}([s, t],[0,+\infty))$, and let $1 / p+1 / q=1$. If $p>1$, then, for any of the positive numbers $a, b$, or $c$, the inequality

$$
\frac{\left(a+c \int_{s}^{t} h(x) k^{p}(x) \Delta x\right)^{1 / p}}{b+c \int_{s}^{t} h(x) k(x) g(x) \Delta x} \leq \frac{\left(a+c \int_{s}^{t} h(x) f^{p}(x) \Delta x\right)^{1 / p}}{b+c \int_{s}^{t} h(x) f(x) g(x) \Delta x}
$$

holds, where $k(x)=(\operatorname{ag}(x) / b)^{q / p}$. The sign of inequality in (39) is reversed if $0<p<1$.

\section{Acknowledgments}

This work was supported by the NNSF of China (Grant no. 61073121), the Natural Science Foundation of Hebei Province of China (Grant no. F2012402037), the Natural
Science Foundation of Hebei Education Department (Grant no. Q2012046), and the Fundamental Research Funds for the Central Universities (Grants nos. 11ML65 and 13ZL). The authors would like to express their sincere thanks to the anonymous referees for their great efforts to improve this paper.

\section{References}

[1] S. Abramovich, J. Pečarić, and S. Varošanec, "Sharpening Hölder's and Popoviciu's inequalities via functionals," The Rocky Mountain Journal of Mathematics, vol. 34, no. 3, pp. 793-810, 2004.

[2] B. Ivanković, J. Pečarić, and S. Varošanec, "Properties of mappings related to the Minkowski inequality," Mediterranean Journal of Mathematics, vol. 8, no. 4, pp. 543-551, 2011.

[3] B. Liu, "Inequalities and convergence concepts of fuzzy and rough variables," Fuzzy Optimization and Decision Making, vol. 2, no. 2, pp. 87-100, 2003.

[4] L. Nikolova and S. Varošanec, "Refinements of Hölder's inequality derived from functions $\psi_{p, q, \lambda}$ and $\varphi_{p, q, \lambda}$, , Annals of Functional Analysis, vol. 2, no. 1, pp. 72-83, 2011.

[5] S. M. Buckley and P. Koskela, "Ends of metric measure spaces and Sobolev inequalities," Mathematische Zeitschrift, vol. 252, no. 2, pp. 275-285, 2006.

[6] I. Franjić, S. Khalid, and J. Pečarić, "Refinements of the lower bounds of the Jensen functional," Abstract and Applied Analysis, vol. 2011, Article ID 924319, 13 pages, 2011.

[7] G. H. Hardy, J. E. Littlewood, and G. Pólya, Inequalities, Cambridge University Press, 2nd edition, 1952.

[8] S. Hencl, P. Koskela, and X. Zhong, "Mappings of finite distortion: reverse inequalities for the Jacobian," The Journal of Geometric Analysis, vol. 17, no. 2, pp. 253-273, 2007.

[9] K. Hu, "On an inequality and its applications," Scientia Sinica, vol. 24 , no. 8, pp. 1047-1055, 1981.

[10] R. Jiang and P. Koskela, "Isoperimetric inequality from the Poisson equation via curvature," Communications on Pure and Applied Mathematics, vol. 65, no. 8, pp. 1145-1168, 2012.

[11] J. Kuang, Applied Inequalities, Shandong Science and Technology Press, Jinan, China, 4th edition, 2010.

[12] J. Mićić, Z. Pavić, and J. Pečarić, “Extension of Jensen's inequality for operators without operator convexity," Abstract and Applied Analysis, vol. 2011, Article ID 358981, 14 pages, 2011.

[13] J. Tian, "Reversed version of a generalized sharp Hölder's inequality and its applications," Information Sciences, vol. 201, pp. 61-69, 2012.

[14] J. Tian, "Inequalities and mathematical properties of uncertain variables," Fuzzy Optimization and Decision Making, vol. 10, no. 4, pp. 357-368, 2011.

[15] J. Tian, "Extension of Hu Ke's inequality and its applications," Journal of Inequalities and Applications, vol. 2011, article 77, 2011.

[16] J. Tian, "Property of a Hölder-type inequality and its application," Mathematical Inequalities \& Applications. In press.

[17] S. Varošanec, "A generalized Beckenbach-Dresher inequality and related results," Banach Journal of Mathematical Analysis, vol. 4, no. 1, pp. 13-20, 2010.

[18] S. H. Wu, "Generalization of a sharp Hölder's inequality and its application," Journal of Mathematical Analysis and Applications, vol. 332, no. 1, pp. 741-750, 2007. 
[19] E. F. Beckenbach and R. Bellman, Inequalities, Springer, Berlin, Germany, 1983.

[20] J. Aczél, "Some general methods in the theory of functional equations in one variable. New applications of functional equations," Uspekhi Matematicheskikh Nauk, vol. 11, no. 3, pp. 3-68, 1956 (Russian).

[21] T. Popoviciu, “On an inequality," Gazeta Matematica şi Fizica A, vol. 11, pp. 451-461, 1959 (Romanian).

[22] J. Tian, "Reversed version of a generalized Aczél's inequality and its application," Journal of Inequalities and Applications, vol. 2012, article 202, 2012.

[23] J. Tian and S. Wang, "Refinements of generalized Aczel's inequality and Bellman's inequality and their applications," Journal of Applied Mathematics, vol. 2013, Article ID 645263, 6 pages, 2013.

[24] P. M. Vasić and J. E. Pečarić, "On the Hölder and some related inequalities," Mathematica, vol. 25, no. 1, pp. 95-103, 1983.

[25] C.-L. Wang, "Characteristics of nonlinear positive functionals and their applications," Journal of Mathematical Analysis and Applications, vol. 95, no. 2, pp. 564-574, 1983.

[26] M. Anwar, R. Bibi, M. Bohner, and J. Pečarić, "Integral inequalities on time scales via the theory of isotonic linear functionals," Abstract and Applied Analysis, vol. 2011, Article ID 483595, 16 pages, 2011.

[27] X. He and Q.-M. Zhang, "Lyapunov-type inequalities for some quasilinear dynamic system involving the $\left(p_{1}, p_{2}, \ldots, p_{m}\right)$ Laplacian on time scales," Journal of Applied Mathematics, vol. 2010, Article ID 418136, 10 pages, 2011.

[28] S. Hilger, "Analysis on measure chains-a unified approach to continuous and discrete calculus," Results in Mathematics, vol. 18, no. 1-2, pp. 18-56, 1990.

[29] S. H. Saker, "Some new inequalities of Opial's type on time scales," Abstract and Applied Analysis, vol. 2012, Article ID 683136, 14 pages, 2012. 


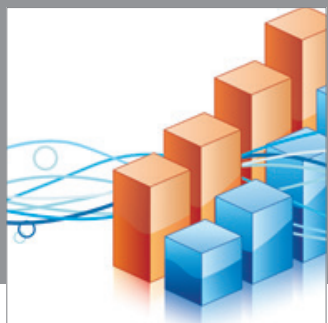

Advances in

Operations Research

mansans

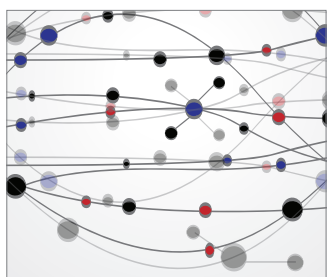

The Scientific World Journal
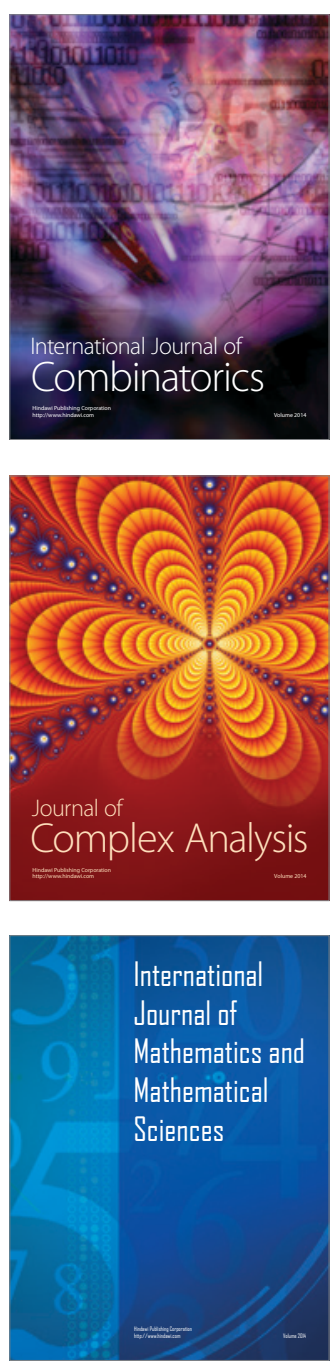
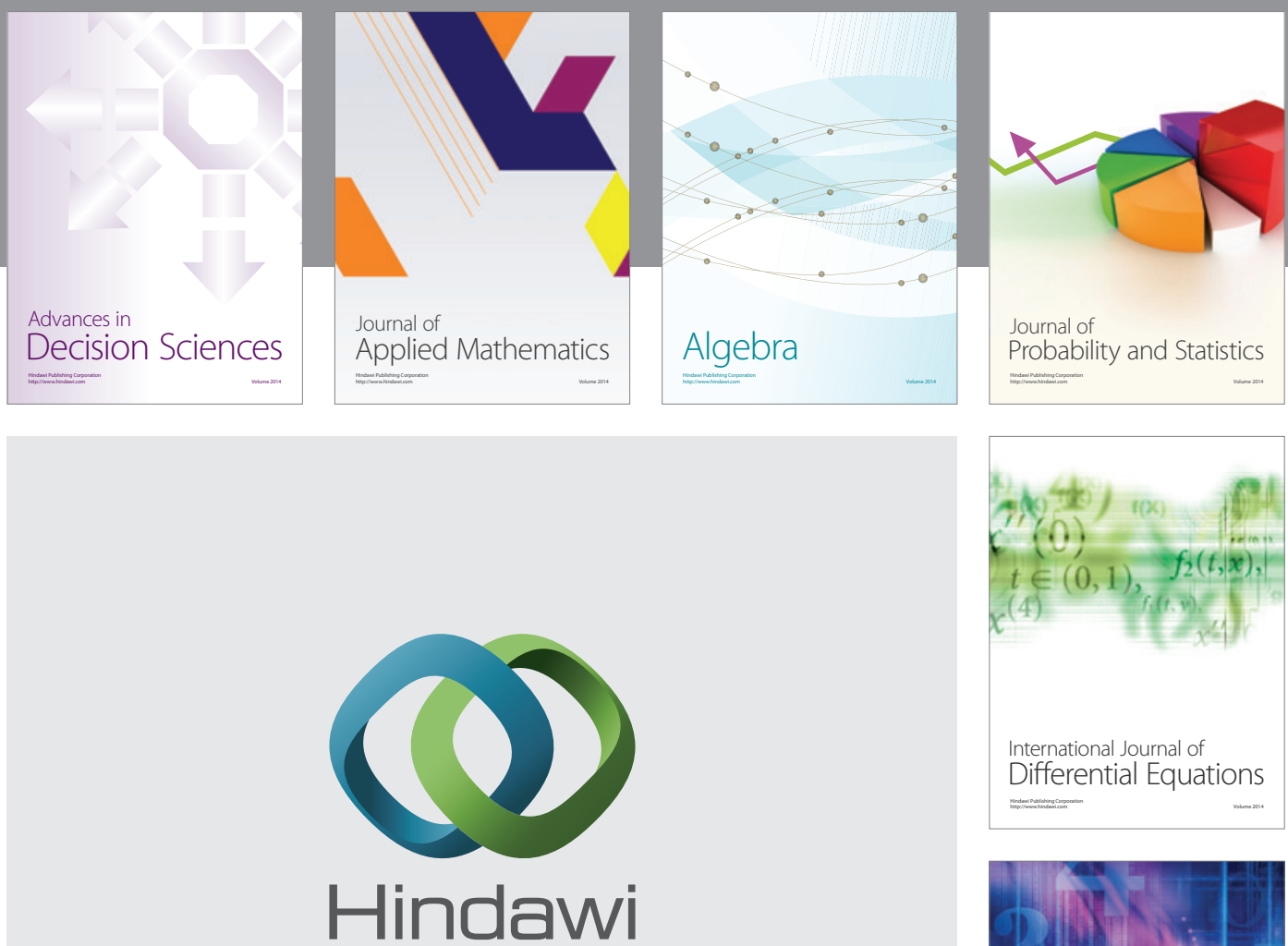

Submit your manuscripts at http://www.hindawi.com
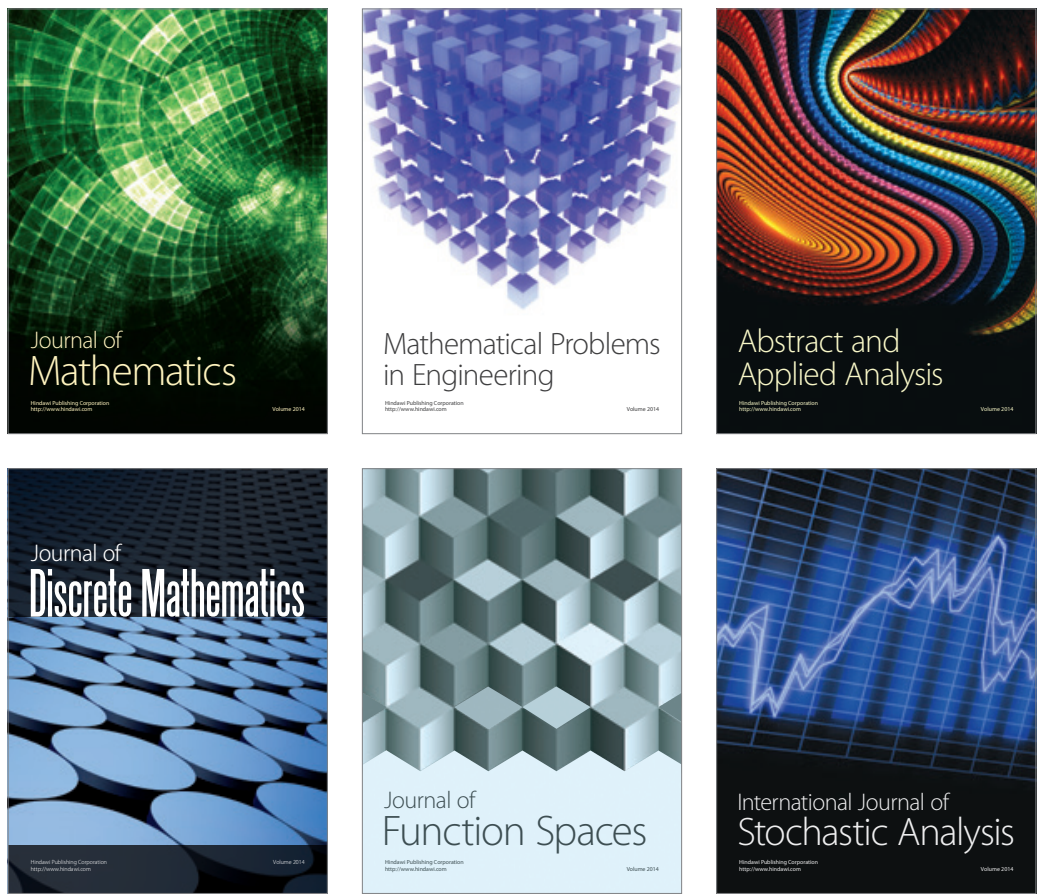

Journal of

Function Spaces

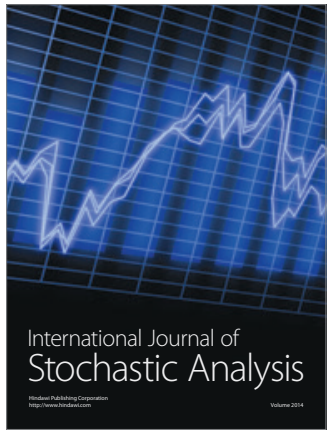

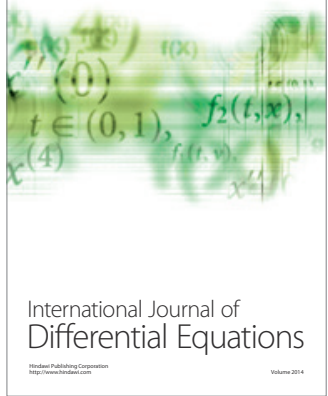
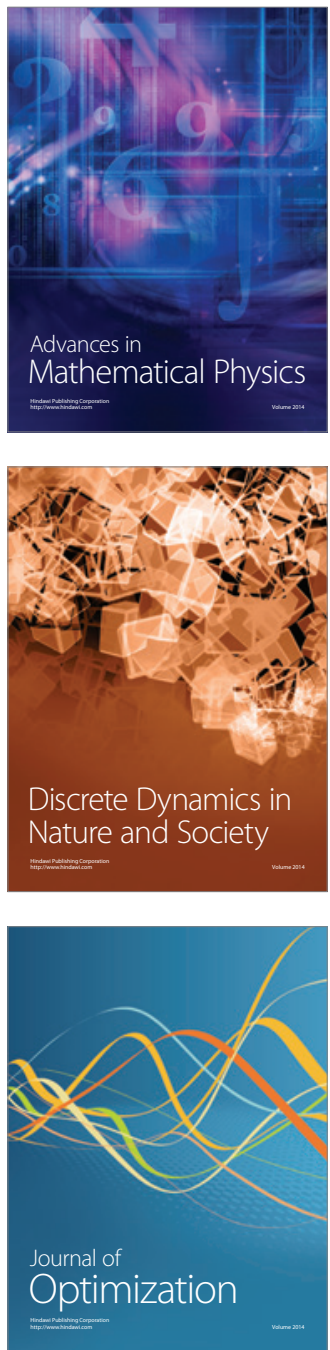